\title{
Transient synovitis of the hip : is systematic radiological screening necessary for the detection of Perthes disease?
}

\author{
Charles Edouard Heylen, Pierre-Louis DocQuier, Dana Dumitriu
}

From the Department of Radiology, Cliniques Universitaires Saint Luc, Université Catholique de Louvain, Brussels, Belgium

\begin{abstract}
Current imaging guidelines in Belgium advise a systematic X-ray screening of the hips after an episode of transient synovitis of the hip, in order to detect Perthes disease. The aim of this study was to analyze whether systematic radiological screening is necessary for all children or whether the X-ray indication could be guided by clinical symptoms.

A retrospective single center study including all children with the diagnosis of transient synovitis of the hip between 2013 and 2018 was performed. 242 patients with the diagnosis of one or more transient synovitis episodes were included, 102 of whom underwent a follow up X-ray. Persistence or recurrence of symptoms were recorded for all patients, as well as the results of follow-up hip X-rays. 12 children did not remain symptom-free after the episode of transient synovitis. Of these patients 10 had a normal follow-up X-ray and 3 were diagnosed with Perthes disease. 1 patient of those 3 had a normal $\mathrm{X}$-ray but was diagnosed with Perthes disease on MRI. Of the children which remained symptom-free after the episode of transient synovitis, none were diagnosed with Perthes disease afterwards.

A follow-up X-ray to exclude Perthes disease after a diagnosis of transient hip synovitis appears to be necessary only in patients with persistent or recurrent symptomatology.
\end{abstract}

Keywords : transient synovitis ; Perthes disease ; X-ray ; hip.

No benefits or funds were received in support of this study. None of the authors have a conflict of interest.

\section{INTRODUCTION}

The most common hip joint disorder in children is transient synovitis (commonly known as irritable hip or toxic synovitis) $(1,2)$. Transient synovitis of the hip (TSH) is a relatively common and benign disease in children, with an average annual incidence of $0.2 \%$ in the general population and a cumulative risk of $3 \%$ of having at least one episode (3). It is more common in boys, with an average sex ratio of 1.7-2.8:1 (3-5). The disease usually affects children between the ages of 3 and 8 years (6-8). The most common symptoms are pain and limited movement

Charles Edouard Heylen',

Pierre-Louis Dovquier ${ }^{2,3}$

- Dana Dumitriu ${ }^{1}$

${ }^{1}$ Department of Radiology, Cliniques Universitaires Saint

Luc, Université Catholique de Louvain, Avenue Hippocrate 10, B-1200 Brussels, Belgium.

${ }^{2}$ Université catholique de Louvain, Secteur des Sciences de la Santé, Institut de Recherche Expérimentale et Clinique, Neuro Musculo Skeletal Lab (NMSK), Avenue Mounier 53, B-1200 Brussels, Belgium.

${ }^{3}$ Department of Orthopedics and Traumatology, Cliniques universitaires Saint Luc, Avenue Hippocrate 10, B-1200 Brussels, Belgium.

Correspondence: Charles Edouard Heylen, Department of Radiology, Cliniques Universitaires Saint Luc, Université catholique de Louvain, Avenue Hippocrate 10, 1200, Brussels, Belgium.

E-mail : charles-edouard.heylen@uclouvain.be

- 2021, Acta Orthopædica Belgica.

Acta Orthopædica Belgica, Vol. 87 - 2 - 2021 
of the hip, as well as refusal to bear weight, most often associated with limping (3). The cause of these symptoms is the presence of a reactive effusion or swelling of the synovium, leading to an analgesic position of the hip in flexion and external rotation.

TSH is a spontaneously resolving non-infectious disease : the swelling of the hip joint heals completely, without causing permanent damage. Although TSH is the most common cause of hip joint effusion in children, other causes of hip inflammation must be excluded, based on history, clinical presentation and blood work, as well as imaging (3,4,9-13).

For the detection of effusion and synovial swelling of the hip, ultrasound (US) is considered the best non-invasive technique (14) but cannot differentiate TSH from other causes of joint effusion such as septic arthritis (4,12,15-18) . X-ray of the hips, if performed in TSH, is normal.

A less common hip pathology, but present in the same age group, is avascular necrosis of the proximal femoral epiphysis, commonly known as Perthes disease (PD). Perthes disease occurs especially in children between 3 and 9 years old, with a peak frequency around 5 years and it is much more common in boys, with a sex ratio of 5:1 (19). The incidence is around 5 in 100,000 children $(19,20)$. In $15 \%$ of cases, it affects both hips but not necessarily at the same time $(19,20)$.

The onset of symptoms in PD is insidious and can mimic TSH (19). A progressive dodge limp sets in, most often painless at the initial stage (1 to 3 months) (21). If present, the pain is mechanical, localized at the hip with radiation to the knee, but it can sometimes be localized only at the knee. Generally, the symptomatology lasts for several months before the diagnosis of Perthes disease is established $(18,22,23)$ and up to three months according to certain authors before being confirmed radiologically (24). Ultrasound at the initial stage may demonstrate synovial tumefaction or synovial effusion similar to transient synovitis. On X-ray, the first signs appear only after several weeks (20) and show a necrosis of the superior femoral epiphysis. Pin hole bone scan and MRI are more sensitive techniques in the early stages of the disease (25). Treatment for Perthes disease is variable (conservative or surgical) depending on the stage of the disease and the age of the patient. Its aim is obtaining the least deformed femoral head possible at the age of bone maturity, in order to prevent secondary coxarthrosis $(20,26)$.

Because early PD can mimic TSH, current Belgian good practice recommendations advise to perform an X-ray of the hips 6 weeks after every episode of TSH, in order to detect PD (27). However, the overwhelming majority of hip X-rays performed following these guidelines are normal and may represent unnecessary radiation exposure for children. The aim of this study was to analyze whether systematic radiological screening after TSH is necessary for all patients or whether the X-ray indication could be guided by clinical evaluation of symptoms. Our hypothesis is that children who later develop PD will have short-term persistence or recurrence of symptoms after an initial episode resembling TSH and that children who become asymptomatic after TSH do not require X-ray follow-up.

\section{MATERIALS AND METHODS}

This retrospective study was approved by the hospital ethics committee and informed consent was waivered. All patients under the age of 16 years who presented one or more episodes of transient synovitis of the hip diagnosed by ultrasound over a period of 5 years (2013-2018) were identified retrospectively.

The inclusion criteria were the presence of synovial thickening and/or hip joint effusion on US, compatible with TSH.

Upon review of their medical files, all cases of septic arthritis, systemic inflammatory arthritis were excluded, as well as patients with haemophilia or those examined in an oncologic or a post-traumatic setting. US examinations were carried out by different operators, both senior pediatric radiologists and radiology interns. All examinations were performed with high-frequency linear transducers, with frequencies between 5 and $18 \mathrm{MHz}$.

Both hips were examined in all cases, in an anterior oblique sagittal section parallel to the long axis of the femoral neck. (Fig. 1) The examiner reported on the presence of synovial swelling, with 


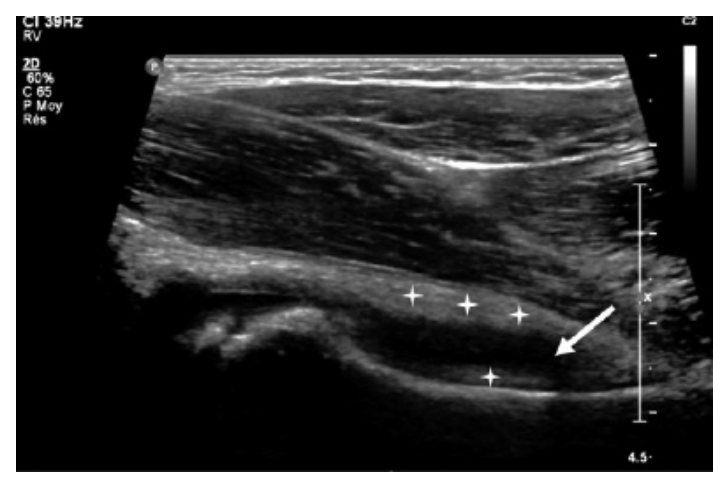

Fig 1. - Anterior oblique sagittal ultrasound section parallel to the long axis of the femoral neck illustrating a synovial tumefaction (stars) of the hip with anechoic joint effusion (arrow).

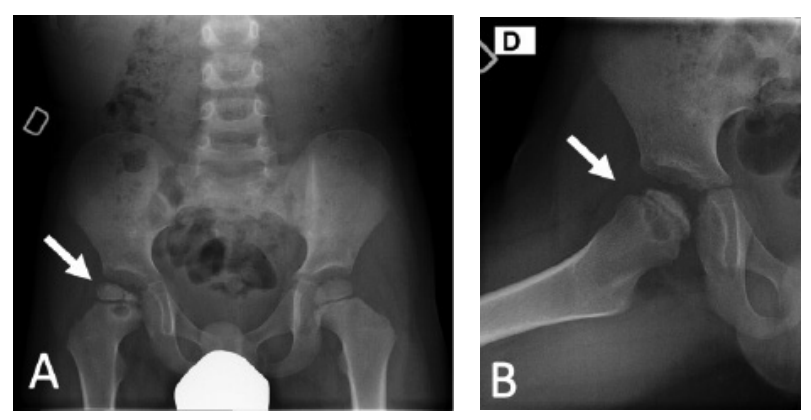

Fig. 2. - Antero-posterior (A) and right lateral (B) X-rays of the hips, demonstrating Perthes disease on the right side (arrow).

or without associated effusion and the thickness of the effusion, if present.

The medical files of the selected patients were reviewed and the following data were collected: child's age at diagnosis, sex, uni- or bilateral synovitis on US, persistent or recurrent symptoms after an episode of TSH, number of episodes of $\mathrm{TSH}, \mathrm{X}$-ray of the pelvis performed after TSH, delay between US and X-ray, diagnosis of Perthes disease on X-ray, diagnosis of Perthes disease by another imaging technique, follow-up after transient synovitis.

The control X-ray of the pelvis was taken in a single antero-posterior view. An additional lateral view of the suspected hip was performed if any abnormality was suspected on the antero-posterior view (Fig. 2).

The sensitivity and specificity, as well as the negative and positive predictive values, of the clinical symptomatology after a suspected episode of transient synovitis for the diagnosis of Perthes disease were calculated.

\section{RESULTS}

242 patients were included in the study, with a total of 268 episodes of TSH. There were 160 boys (66\%) and 82 girls (34\%) (ratio $1.95: 1$ ). 21 patients presented more than one episode of TSH.

The average age of the patients included was 4 years and 3 months, with a minimum age of 1 year, maximum of 12 years, and a median of 4 years.

On US, hip involvement was bilateral in 146 patients $(60 \%)$ and unilateral in $96(40 \%)$.

140 patients $(57.9 \%)$ did not have follow-up by pelvic X-ray. Of these, 81 were followed-up in the orthopedics consultation and 59 were not. The medical record review did not identify any cases of Perthes disease for this group of patients.

102 patients $(42.1 \%)$ benefited from radiological and orthopedic follow-up. Their clinical course and the diagnosis of Perthes disease according to pelvic $\mathrm{X}$-ray are illustrated in Table 1.

In our study cohort, of the 12 patients with persistent symptoms after suspected TSH, Perthes disease was diagnosed in 3 (specificity $88 \%$, positive predictive value 0.25 ). Two of these were identified by pelvic X-ray (table 1) and one by MRI (patient with a negative $\mathrm{X}$-ray and persistent symptoms).

Table 1. - Results of the follow-up pelvis X-rays after transient synovitis in patients with and without persistent symptoms

\begin{tabular}{|l|c|c|c|}
\hline Persistent symptoms & X-ray positive for Perthes & X-ray negative for Perthes & TOTAL \\
\hline YES & 2 & 10 & 12 \\
\hline NO & 0 & 67 & 67 \\
\hline UNKNOWN & 0 & 23 & 23 \\
\hline & 2 & 100 & 102 \\
\hline
\end{tabular}


None of the patients who became asymptomatic after an episode of TSH were subsequently diagnosed with PD (sensitivity $=100 \%$, negative predictive value $=1$ ).

The average delay between US and pelvic X-ray was 59 days ( 8 weeks and 3 days) and the median 39 days ( 5 weeks and 4 days), with limits between 0 and 684 days.

\section{DISCUSSION}

Transient synovitis of the hip is a frequent pathology and the first cause of hip pain in children aged 4 to 10 years $(1,2)$ with a predominance between the ages of 3 and 8 years (6-8). It is a benign condition with a good prognosis.

On the other hand, Perthes disease is less common but requires an early diagnosis because depending on the duration of symptoms and the stage of the disease's development, treatment and prognosis are different, with risk of developing early secondary coxarthrosis $(20,26)$.

Current Belgian recommendations advise performing a control X-ray of the pelvis 6 weeks after the episode of TSH in order to exclude PD. The main objective of this retrospective study was to determine whether all children with TSH require a follow-up X-ray, regardless of their symptoms.

The results show that in the absence of persistent symptomatology after an episode of TSH, there was no diagnosis of PD by an X-ray of the pelvis or any other technical modality (negative predictive value : 1, Sensitivity : 100\%).

Conversely, in our population, all of the three children with hip joint swelling or effusion hip which turned out to be the first manifestation of PD, presented persistent symptomatology after TSH.

Of the 12 patients with persistent symptomatology, only 3 were diagnosed with Perthes disease (positive predictive value: 0.25 , Specificity : $88 \%$ ). Among these three cases of Perthes disease, only one patient was diagnosed by X-ray 6 weeks post$\mathrm{TSH}$, according to the Belgian guidelines. A second patient did not have an X-ray follow-up after a first episode of TSH, but upon recurrence of symptoms and joint effusion 10 months later, hip X-ray was performed, demonstrating PD. For the third patient
X-ray and US were performed at the same time, 4 weeks after the start of limping and hip pain. The X-ray was considered normal, but given the persistence of symptoms MRI was performed, revealing PD.

Several studies have already been published regarding follow-up after TSH.

A recent Swiss retrospective study published by Lenoir et al. in 2016 produced the same results as our study, with the difference that the control X-ray was performed at 3 months systematically (28). Patient compliance in that study was better than ours, with a total of 198 out of 212 patients who had a control X-ray at 3 months (93\% versus $42 \%$ in our study). Similar to our study, no asymptomatic patient in the course of TSH was diagnosed with PD and all patients with PD experienced persistent or recurrent symptoms.

Another Swiss retrospective study published by Dubois-Ferrière et al. in 2015 corroborates our analyses concerning the usefulness of additional investigations in the monitoring of TSH in asymptomatic children (29).

A systematic review of the literature on the clinical course of transient synovitis by Asche et al. demonstrated that two weeks after an episode of TSH almost all patients were asymptomatic (18). Patients with symptoms lasting more than a month were more often found to be cases of PD, initially considered as TSH. In the long term, 0 to $10 \%$ of children with TSH were diagnosed with PD. On this basis, the authors issued the recommendation that children with TSH should be clinically followedup for at least six months to increase the chances of not missing PD. In the studies included in this systematic review, it is noted that in children with a final diagnosis of PD, the presumed episode of transient synovitis occurred atypically, with persistent or intermittent complaints beyond the normal course of TSH (18). This coincides with the results of our study, as well as those of Lenoir et al (28).

Based on our results, we can confirm that the disappearance of symptoms after an episode of TSH is an excellent factor for excluding PD, with a negative predictive value of $100 \%$ in our population. Therefore, an X-ray of the pelvis at 6 weeks after 
TSH in an asymptomatic patient does not seem appropriate to us.

Unfortunately, it is not possible to make a recommendation regarding the optimal timeframe for performing the hip X-ray post-TSH for symptomatic patients based on this study, for two reasons. On the one hand, the number of positive cases of PD was low in our study, but consistent with the frequency reported in other studies with a similar population size (28). On the other hand, the delay between the episode of TSH and the X-ray of the pelvis was variable in our population, with however most of the controls carried out between 4 and 12 weeks.

The strengths of this study were a population of adequate size, significant and representative of the general pediatric population. The study period of over 5 years allowed enough time for a proper follow-up of patients.

One limitation of the study is the fact that more than half $(57,9 \%)$ of the patients with hip effusion or swelling reported on US did not have a followup X ray, either because it was not requested by the clinician or because of low compliance by the patients. This is largely due to the retrospective nature of the study, but could also mean that some clinicians already consider follow-up X-rays after TSH in asymptomatic patients unnecessary and apply the guidelines selectively. It is important to remember that, although we did not take into account patients without X-ray follow-up in the statistical calculations, no case of Perthes disease was diagnosed in this group, based on the review of their medical files.

\section{CONCLUSION}

Based on the results of this study and recent literature, we were able to confirm our hypothesis that a follow-up X-ray of the hips to exclude Perthes disease after transient synovitis of the hip is necessary only in patients with persistent or recurring symptoms.

\section{REFERENCES}

1. Fabry G. Clinical practice: the hip from birth to adolescence. Eur J Pediatr. 2010 ; 169 : 143-8.
2. Whitelaw CC, Varacallo M. Transient Synovitis. 2020 Jun 27. In : StatPearls (Internet). Treasure Island (FL) : StatPearls Publishing ; 2021.

3. Landin LA, Danielsson LG, Wattsgård C. Transient synovitis of the hip. Its incidence, epidemiology and relation to Perthes' disease. J Bone Joint Surg Br. 1987 ; 69 : 238-42.

4. Fischer SU, Beattie TF. The limping child : epidemiology, assessment and outcome. J Bone Joint Surg Br. 1999 ; 81 : 1029-34.

5. Vijlbrief AS, Bruijnzeels MA, van der Wouden JC, van Suijlekom-Smit LW. Incidence and management of transient synovitis of the hip : a study in Dutch general practice. Br J Gen Pract. 1992 ; 42 : 426-8.

6. Nouri A, Walmsley D, Pruszczynski B, Synder M. Transient synovitis of the hip : a comprehensive review. $J$ Pediatr Orthop B. 2014 ; 23 : 32-6.

7. Zamzam MM. The role of ultrasound in differentiating septic arthritis from transient synovitis of the hip in children. J Pediatr Orthop B. 2006 ; 15 : 418-22.

8. Baskett A, Hosking J, Aickin R. Hip radiography for the investigation of nontraumatic, short duration hip pain presenting to a children's emergency department. Pediatr Emerg Care. 2009 ; 25 : 78-82.

9. Bickerstaff DR, Neal LM, Booth AJ, Brennan PO, et al. Ultrasound examination of the irritable hip. J Bone Joint Surg Br. $1990 ; 72: 549-53$.

10. Konermann W, de Pellegrin M. Die Differentialdiagnose des kindlichen Hüftschmerzes im Sonogramm. Coxitis fugax, Morbus Legg-Calvé-Perthes, Epiphyseolysis capitis femoris (The differential diagnosis of juvenile hip pain in the ultrasonographic picture. Transient coxitis. LeggCalvé-Perthes disease, epiphysiolysis of the femur head). Orthopade. $1993 ; 22: 280-7$.

11. Graf J, Bernd L, Niethard FU, Kaps HP. Die Diagnostik bei der Coxitis fugax, der häufigsten Hüfterkrankung beim Kind (Diagnosis of coxitis fugax, the most frequent hip disease in childhood). Klin Padiatr. 1991 ; 203 : 448-51.

12. Ryan DD. Differentiating Transient Synovitis of the Hip from More Urgent Conditions. Pediatr Ann. 2016; 45 : e209-13.

13. Caird MS, Flynn JM, Leung YL, Millman JE, et al. Factors distinguishing septic arthritis from transient synovitis of the hip in children. A prospective study. $J$ Bone Joint Surg Am. 2006 ; 88 : 1251-7.

14. Gopakumar TS, Vaishya R, Klenerman L, Carty H. The role of ultrasound and isotope scanning in the management of irritable hips. Eur J Radiol. 1992 ; 15 : 113-7.

15. Cook PC. Transient synovitis, septic hip, and Legg-CalvePerthes disease: an approach to the correct diagnosis. Pediatr Clin North Am. 2014 ; 61 : 1109-18.

16. Houghton KM. Review for the generalist : evaluation of pediatric hip pain. Pediatr Rheumatol Online, $2009 ; 7: 10$.

17. Yagupsky P. Differentiation between septic arthritis and transient synovitis of the hip in children. J Bone Joint Surg Am. 2005 ; 87 : 459 ; author reply 459-60. 
18. Asche SS, van Rijn RM, Bessems JH, Krul M, et al. What is the clinical course of transient synovitis in children : a systematic review of the literature. Chiropr Man Therap. $2013 ; 21: 39$.

19. Wenger DR, Ward WT, Herring JA. Legg-Calve-Perthes disease. J Bone Joint Surg Am. 1991 ; 73 : 778-88.

20. Hariga H, Docquier PL. La maladie de Legg-CalvéPerthès. In : X Banse, O Barbier, P-L Docquier (ss dir), Manuel pratique d'orthopédie et de traumatologie, 2015 ; $1-7$.

21. Mills S, Burroughs KE. Legg Calve Perthes Disease. 2020. In : StatPearls (Internet). Treasure Island (FL) : StatPearls Publishing ; 2021.

22. Chaudhry S, Phillips D, Feldman D. Legg-Calve-Perthes disease: an overview with recent literature. Bull Hosp Jt Dis. 2013 ; 72 :18-27.

23. Ceroni D, Kaelin A. The Legg-Calve-Perthes disease: which assessment? Which therapeutic approach? Rev Med Suisse. $2006 ; 2$ : 2908-12.

24. Dimeglio A, Canavese F. Imaging in Legg-Calve-Perthes disease. Orthop Clin North Am 2011 ; 42 : 297-302.
25. Conway JJ. A scintigraphic classification of Legg-CalvePerthes disease. Semin Nucl Med. 1993 ; 23 : 274-95.

26. Joseph B, Price CT. Consensus statements on the management of Perthes disease. Orthop Clin North Am. 2011 ; 42 : 437-40.

27. SPF Recommandations pour l'usage correct de l'imagerie médicale (Online). Brussels (Belgium): SPF Santé Publique; 2010. SPF Recommandations imagerie médicale (modified on $01 / 10 / 2010$; cite the $01 / 06 / 2021$; (about 1 screen). Available at : https://www.health.belgium.be/fr/ sante/organisation-des-soins-de-sante/qualite-des-soins/ bonnes-pratiques/recommandations-imagerie.

28. Lenoir U, Slongo T, Aghayev E, Joeris A. The Value of Conventional Radiographs of the Pelvis in Detection of Perthes Disease 3 Months After an Episode of Acute Transient Synovitis. Klin Padiatr. 2017 ; 229 : 76-81.

29. Dubois-Ferriere V, Belaieff W, Lascombes $P$, de Coulon $\mathbf{G}$, et al. Transient synovitis of the hip: which investigations are truly useful? Swiss Med Wkly. 2015 ; 145 : w14176. 